NDLisis:

\title{
THE HISTOGENESIS OF FOUR CASES OF ANGIOMYXOMA OF THE AURICLE
}

\author{
BY \\ C. RAEBURN \\ From the Area Pathological Laboratory, Whipps Cross Hospital, Essex
}

(RECEIVED FOR PUBLICATION MAY 22, 1952)

Many individual primary tumours of the heart have now been reported and there are two general reviews in the English literature, those of Yater (1931) and Prichard (1951), and also an extensive monograph in French by Mahaim (1945). The addition of a few more would be of little interest, but the study of 10 benign tumours, collected from some 19,000 necropsies performed by the staff of a central histological laboratory, has yielded facts of importance in the aetiology and designation of these lesions. Four of these tumours were angiomyxomas arising in the left auricle and they alone are considered in this paper.

\section{Technique}

Portions of tissue were fixed in formol saline and embedded in paraffin wax. The following stains and histochemical methods were employed; haematoxylin and eosin, iron haematoxylin and Van Gieson, Heidenhain's iron haematoxylin, Mallory's phosphotungstic acid haematoxylin, and Weigert's stain for elastic fibres. Mucin was demonstrated by Southgate's method, by toluidine blue, and by the periodicacid-Schiff reaction (P.A.S.). The last two methods were also used after prior treatment of the sections with hyaluronidase. Best's method was employed as well as P.A.S. to demonstrate glycogen. Iron was demonstrated by the method of Perls. Frozen sections of one myxoma were stained by Sudan III for fat.

\section{Case Reports}

Positive clinical findings were available for one case ; the remaining three were apparently free of cardiac symptoms. In two instances the myxoma was undoubtedly responsible for death.

Case 1 (406/51). - A man aged 68 Complained of attacks of shortness of breath, cough, pain in the chest and swelling of the legs over a period of a year. The physical signs were those of intermittent congestive heart failure with bilateral pleural effusions. There were no cardiac murmurs but auricular flutter and partial heart block occurred at intervals (Fig. 1). After many relapses and recoveries he died suddenly during an attack of congestive heart failure. The body was that of a well-nourished but oedematous man. The general visceral findings may be summarized as those of prolonged right heart failure in every way comparable with those due to mitral stenosis. There were small pleural effusions and the liver and spleen were enlarged. The lungs contained multiple infarcts, and organizing thrombi were present in many of the intrapulmonary branches of the pulmonary artery. The right ventricle and the left auricle were greatly dilated and the latter contained a pear-shaped mass $(4.5 \times 3.8 \times 3.5 \mathrm{~cm}$. $)$ wh:ch was attached by a short, flattened pedicle to the lower border of the fossa ovalis and depended into the mitral orifice in such a manner as to maintain the valve cusps in the half-open position (Fig. 2). The mass was of elastic consistency and the external surface, mainly smooth but with some areas of velvety texture, was deep red with a mottling of yellow. The

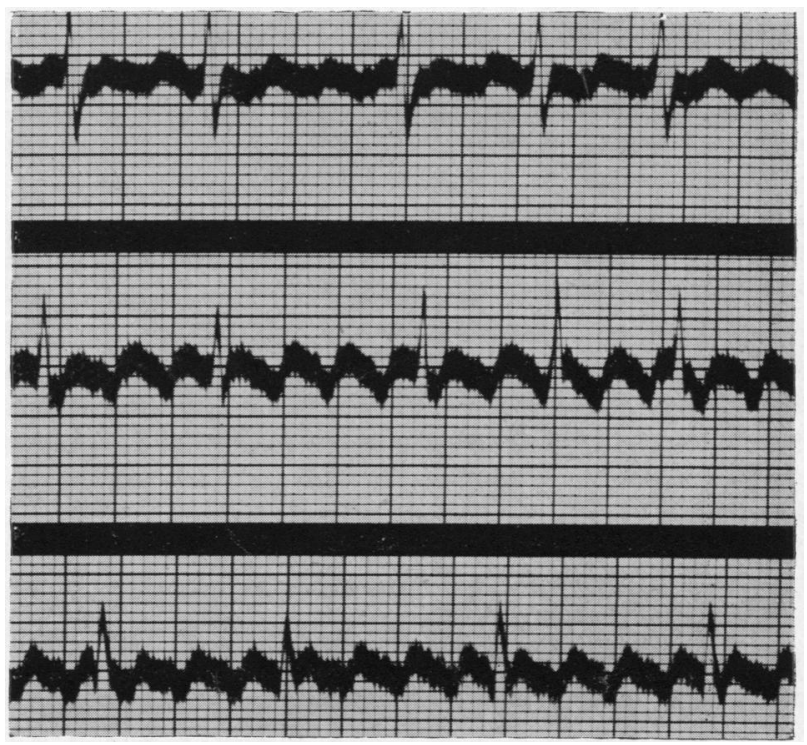

FIG. 1.-Electrocardiogram showing auricular flutter and partial heart b.ock due to myxoma of left auricle. 


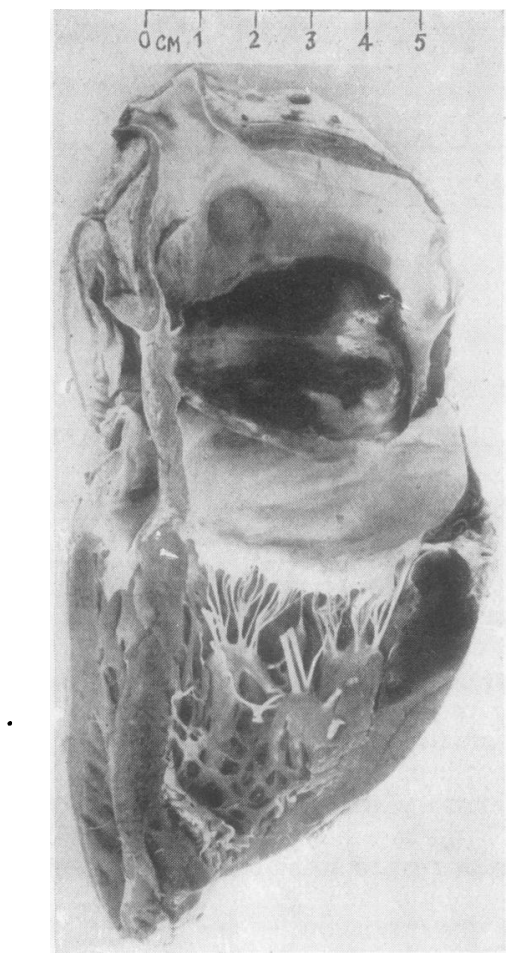

FIG. 2-Cross section of myxoma of left auricle. The auricle $i$ hypertrophied, the mitral cusp and chordae tendineae are slightly thickened.

cut surface was composed of soft, yellow tissue with haemorrhagic areas. The mitral valve cusps and the chordae tendineae were slightly and uniformly thickened, but without distortion or fusion of the margin of the cusps, and the left auricle was hypertrophied to a thickness of $0.6 \mathrm{~cm}$. but there was no suggestion of endocarditis. Fragments of ante-mortem thrombus were present in both auricular appendices.

Histology.-The surface of the tumour was covered by endothelium and there were occasional groups of short blunt villi corresponding to the gross areas of velvety texture. Beneath the endothelium was a fibroelastic lamina with numerous breaches of continuity where the elastic fibres ended in hyalinized collagen bundles or had become separated and ultimately lost in structureless areas. Towards the pedicle the elastic lamina was intact and it fused with the superficial fibres of the elastica of the auricle, which thus divided to enclose the tumour (Fig. 3). Formed blood vessels were confined to the pedicle but capillaries and large vascular spaces lined by endothelium were plentiful. Stromal haemorrhages were numerous and iron pigment was diffusely scattered. The bulk of the tumour was composed of mucinous material in which collagen and elastic fibres ramified irregularly. Lying in groups, strands, and in branching formations in the myxoma- tous stroma were the lepidic* cells characteristic of $\stackrel{\mathscr{0}}{5}$ auricular myxoma (Fig. 4). Many of. these cells re- 을 sembled swollen and rounded fibroblasts which were $\vec{F}$. grouped into slender solid cords, but in some strands $\stackrel{\sigma}{+}$ a lumen, in which red cells were occasionally present, $\bar{O}$ had appeared (Fig. 4). Every transition could be 므 found from solid cords through primitive vascular $\bar{m}$ channels to large, irregular vascular spaces lined in $\widehat{\nabla}$ part by cubical or fusiform cells and in part by flat- 응 tened endothelium. This was a classical "myxoma" is but its essentially angiomatous nature was readily $\overrightarrow{0}$ apparent. When sections were treated with toluidine blue small areas of metachromatic staining appeared $\vec{\omega}$ at random in the matrix and particularly in the region 2 of the large vessels in and near the pedicle. This metachromatic substance was removed by treatment or with hyaluronidase. The matrix as a whole stained $\rightarrow$ well by Southgate's mucicarmine and the P.A.S. technique and this mucin was not digested by $\mathcal{O}$ hyaluronidase. Glycogen and fat were absent.

Case 2 (1076/37). - A woman aged 78, with no his- $\rightarrow$ tory of cardiac or pulmonary disease, died from gan- $z$ grene of the rectum. At necropsy a pear-shaped, slightly lobulated mass $(5 \times 3 \times 2.5 \mathrm{~cm}$. $)$ was found arising from the lower border of the fossa ovalis and $\underset{\mathbb{D}}{ }$ depending through the mitral orifice by which it was slightly constricted. The tumour was of rubbery con- $\vec{\varphi}$

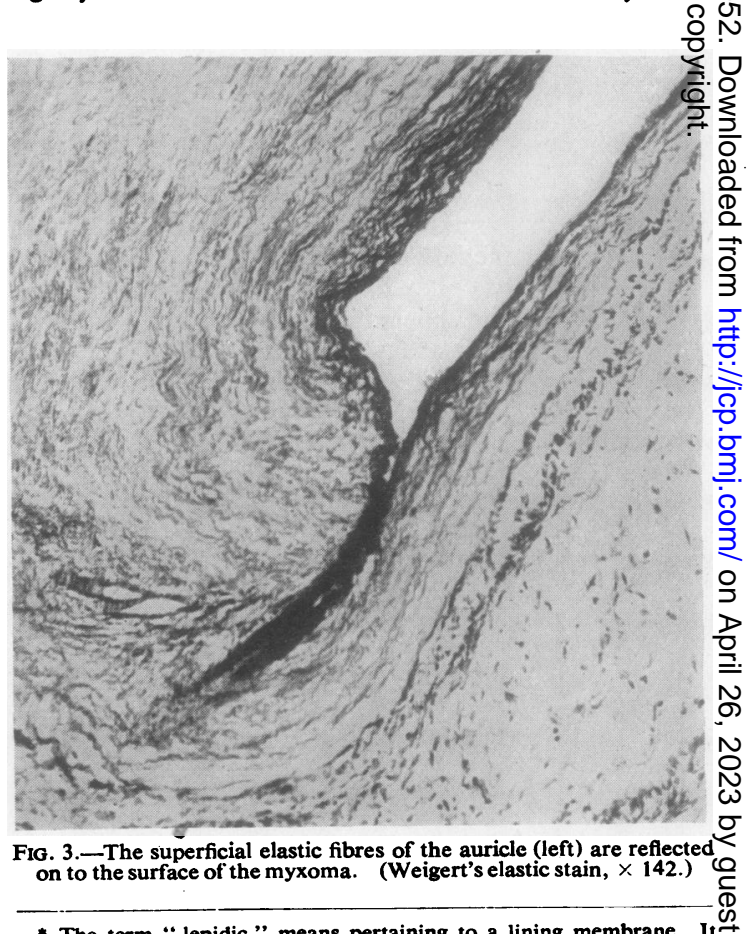

*The term " lepidic" means pertaining to a lining membrane. It:has for long been applied to the principal cells of these tumours and $\square$ is justified as there is a continual evolution of solid cords of cells $\bar{O}$ and eventually of vascular spaces. The fibroblast is regarded as the precursor of the lepidic cell which is the precursor of the endothelial cell.

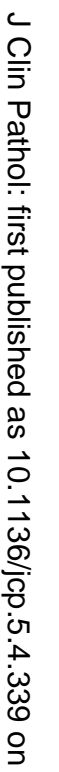


sistency and mottled red and yellow on both the external and the cut surfaces. The cusps of the mitral valve and the chordae tendineae were slightly and uniformly thickened but there was no evidence of endocarditis.

Histology.-The tumour was covered by endothelium and beneath this there was a condensation of elastic fibres which increased in thickness towards the base and became continuous with the superficial fibres of the auricular elastica. The main bulk of the tumour was composed of structureless mucinous material in which delicate elastic and collagenous fibres ramified. In degenerate areas collagen and elastic fibres were heavily impregnated with iron (Fig. 5). Scattered in the gelatinous matrix were groups and strands of lepidic cells undergoing vascular differentiation and there were a few formed vessels with muscle fibres and an elastic lamina in the region of the pedicle. Large vascular spaces in the matrix were lined by flattened endothelium, with interpolated groups of fibroblast-like cells often several deep, and contained by a single collagen strand. The vasoformative elements were clearly the important histological component. The subjacent auricular elastic tissue was atrophied but the auricular muscle was hypertrophied. The mucinous ground substance took all the mucin stains diffusely and was focally metachromatic with toluidine blue. Hyaluronidase acted only on these metachromatic areas.

Case 3 (526/38). - A man aged 73, with no history of any thoracic disability or pain, died from oedema

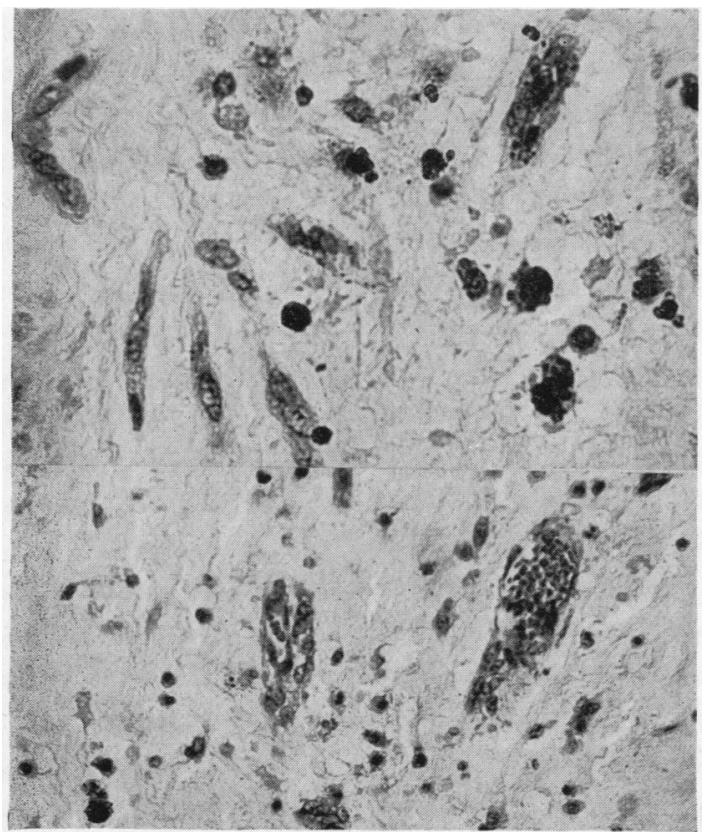

Fig. 4.-Vasoformative lepidic cells in auricular myxoma. (Haematoxylin and eosin. Top, $x 340$, below, $x$ 200.)

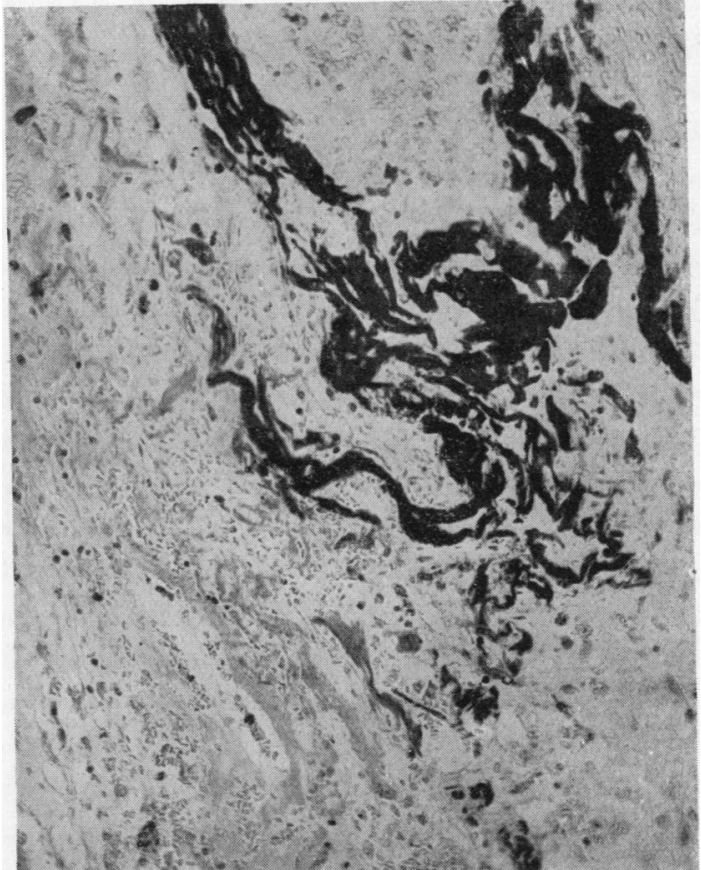

FIG. 5.-Iron impregnation of collagen bundles in auricular myxoma. (Perls' method, $x$. 160.)

of the lungs following prostatectomy. At necropsy an elongated, pear-shaped tumour, similar to those already described and measuring $7.5 \times 5 \times 4 \mathrm{~cm}$.; was found to arise from the posterior border of the fossa ovalis and to extend through the mitral valve, by which it was slightly constricted, to fill most of the cavity of the left ventricle (Fig. 6). The mitral valve cusps were normal but the left ventricle was hypertrophied to a thickness of $1.8 \mathrm{~cm}$.

Histology.-The mass was partly covered by endothelium and partly by a thin lamina of ante-mortem thrombus. Lepidic cells, a few of which contained fine particles of glycogen, were scattered singly and in vasoformative groups in a mucinous stroma containing collagen and elastic fibres and much iron pigment. The tumour vessels were mainly large, endothelium-lined spaces, each bounded by a single collagen fibre but towards the base there were formed vessels with a muscular coat and an elastic lamina. Elastic fibres were condensed peripherally in the region of the angle between the tumour and the auricle and the superficial fibres of the auricular elastica were continued into the periphery of the tumour until they vanished into areas of necrosis. Beneath the base of the tumour the elastic tissue of the auricle had atrophied and foci of lepidic cells were mingled with the auricular muscle fibres.

Case $4(1466 / 40)$. - This lesion was an incidental post-mortem finding in a man aged 69 dying of bronchopneumonia associated with a buccal carci- 


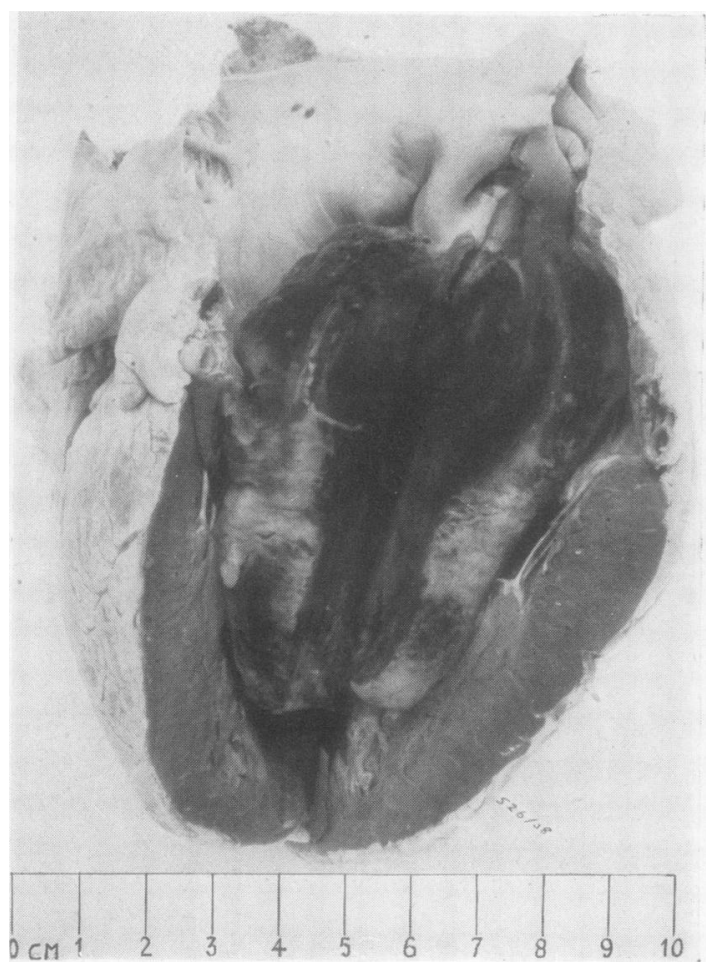

FIG. 6. - A large myxoma of the left auricie which passes through the mitral valve and fills the cavity of the left ventricle.

noma. A slightly lobulated, firm, dark red nodule $1.5 \mathrm{~cm}$. in diameter projected into the left auricle immediately below the lower border of the fossa ovalis. The cut surface was composed of red, spongy tissue enclosed by a fibrous capsule $0.1 \mathrm{~cm}$. in thickness.

Histology.-The tumour was a capillary and cavernous haemangioma. The smaller vascular spaces were enclosed by swollen endothelial cells, the larger having, in addition, a wall composed of delicate, intertwined collagen fibres separated in places by a slightly mucinous matrix, and there were occasional small groups of cells of lepidic appearance. Iron pigment was scattered in coarse, granular aggregates and collagen and elastic fibres were often impregnated with iron. There were several fragments of bone (Figs. 7 and 8). Peripherally condensed elastic fibres could not be traced into continuity with those of the auricle as the auricular elastic tissue beneath and lateral to the angioma was deficient, either primarily, as a congenital hypoplasia, or secondarily as a pressure atrophy. Comparison with the arrangement of the elastic tissue in the myxomas favours hypoplasia.

\section{Auricular Thrombus}

As many still believe that the tumours described are no more than organized thrombi a brief

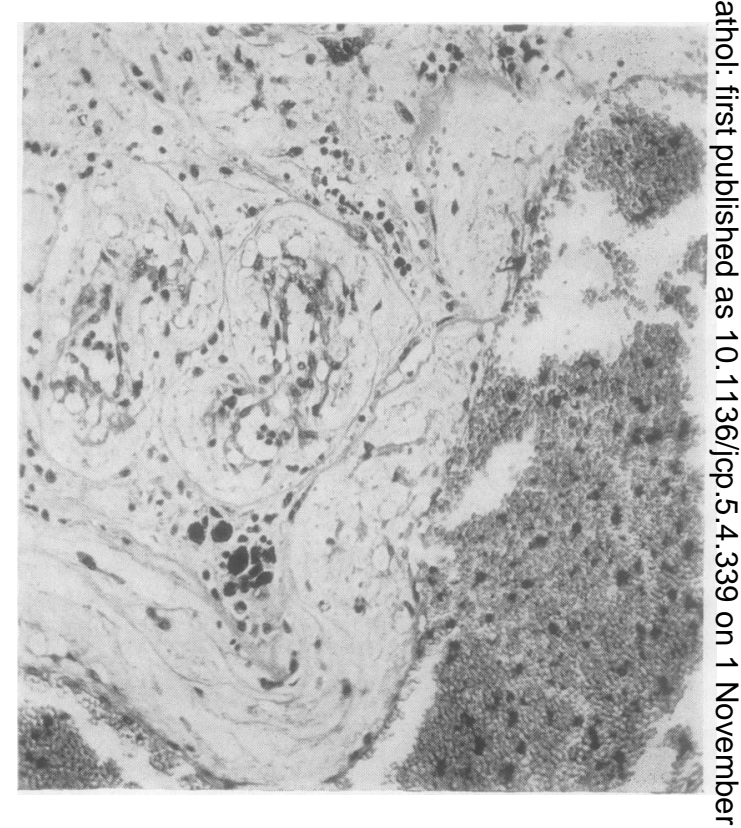

FIG. 7.-Auricular angioma; there are large vascular elements but $\overrightarrow{0}$ the smaller vessels in the mucoid matrix are composed of cellse very like the lepidic cells of the myxoma. (Haematoxylin adr eosin, $\times 140$.)

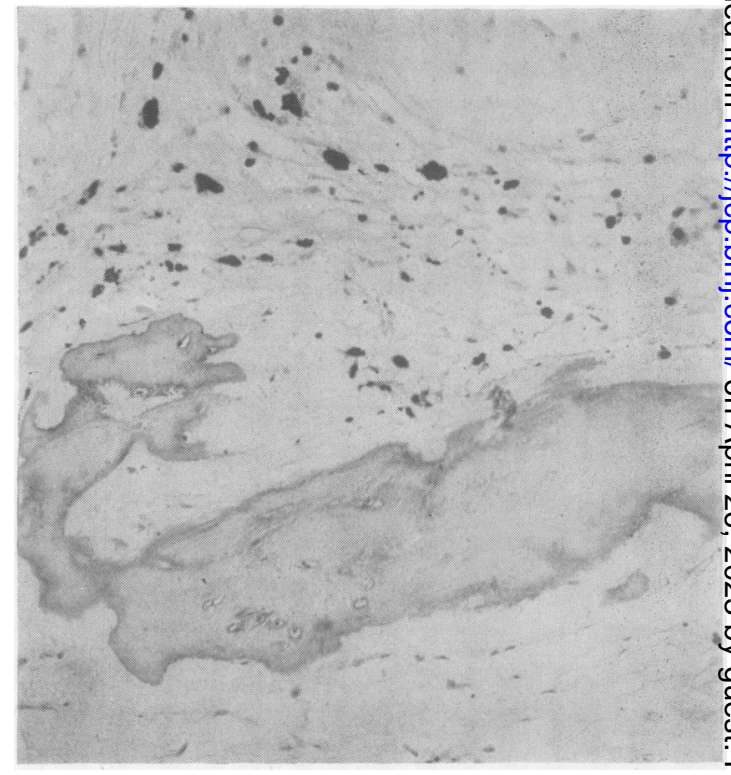

Fig. 8.-Auricular angioma; bone formation in a mucoid matrix containing much free iron. (Perls' method. Neutral red counterstain, $\times 120$.) 
account of thrombi is relevant to the discussion. Auricular thrombi are frequently found at necropsy and some show organization of their mural aspect, but a study of 30 such thrombi has failed to demonstrate the slightest resemblance to the angiomyxomas. A pedunculated thrombus in the right auricle does, however, merit description on account of its situation and outward form.

Case Report.-A woman aged 57, known to have suffered for many years from rheumatic heart disease, died from congestive heart failure. At necropsy the heart was greatly enlarged by dilatation and hypertrophy of both auricles and of the right ventricle. There was a moderate degree of mitral stenosis. The right auricle contained an ovoid mass, measuring $2 \times 1 \times 1 \mathrm{~cm}$., attached by a pedicle $0.5 \mathrm{~cm}$. in diameter to the upper border of the fossa ovalis. The mass was firm, reddish ochre, and slightly granular on the surface while the cut surface was ochre, dry, and rather friable with a suggestion of lamination of the outer portion.

Histology.-The greater part of the mass was composed of structureless material devoid of endothelial covering. A zone at the base was invaded by fibroblasts and capillaries and was typical of the granulation tissue of an organizing thrombus (Fig. 9). The only elastic fibres were those associated with formed vessels in and near the pedicle. Metachromatic stromal mucin was present in the amount normally associated with granulation tissue but there was no other mucinous substance. Iron pigment was scanty and confined to histiocytes in the organized area. The elastic lamina of the auricle was intact beneath the thrombus.

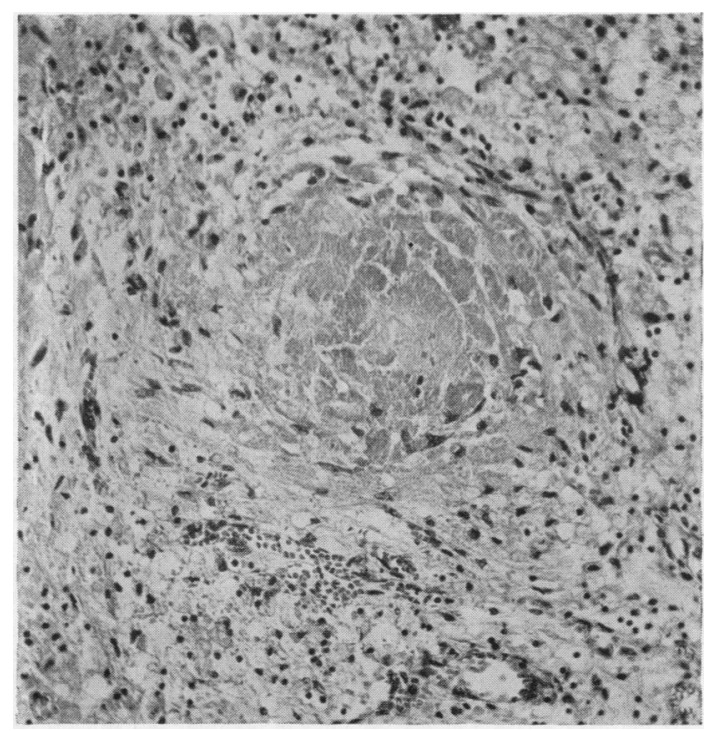

FIG. 9.-The base of an organizing, pedunculated thrombus from the right auricle for comparison with myxoma. (Haematoxylin and eosin, $\times$ 150.)

\section{Discussion}

Myxomas of the left auricle are clinically silent or may present the symptoms of intermittent mitral stenosis with phases of severe right heart failure and occasionally syncopal attacks due to the tumour sinking into the mitral valve. Auscultatory signs are variable but enlargement of the left auricle and of the right ventricle may be demonstrated radiologically. Von Reis (1949) states that the electrocardiogram is characterized by right ventricular preponderance and expansion of the $P$ waves. Rheumatic and other forms of endocarditis must be excluded.

The four tumours described are all variants of the same intrinsic structure. The first is the best example of the myxoma and the last is an angioma with slight myxomatous attributes. All arise from the same anatomical site, the rim of the fossa ovalis, and all are composed of identical structures namely, vasoformative lepidic cell groups, formed vessels, collagenous and elastic fibres, and myxomatous tissue. The nature of the mucin has not been determined. By Southgate's method or by the P.A.S. technique the three myxomas stain well and diffusely and the angioma stains irregularly and weakly. When stained with toluidine blue all the tumours show areas of metachromatic staining, mainly towards the base in the vicinity of formed vessels. Treatment with hyaluronidase removes the metachromatic staining substance but does not affect the diffuse mucin reaction.

The precise nature of these tumours has been the subject of controversy and, as most workers have seen but one or two, there has been a tendency to unfortunate generalization based on incompletely studied cases. Thorel $(1903,1915)$ stated that myxomas were always associated with venous thrombosis and that the histological descriptions were those of organization of a thrombus but he relied upon the accounts of others and had apparently no personal experience. Warthin (1916) described mucoid, fibroblastic tissue in organized cardiac thrombi but his observations remain unconfirmed and they bear no resemblance to the histology of myxoma. Husten (1922) agreed with Thorel after studying what seems to have been an organizing mural thrombus in the right auricle. The thrombus theory is an assumption that in special circumstances a thrombus may simulate a neoplasm both macroscopically and microscopically. Against this theory are the facts that auricular myxomas are never laminated, they are histologically unlike an organized thrombus, they do not occur in the auricular appendices, they are rare while thrombi are common, and no transitional stages between thrombus and myxoma have ever 
been described. If myxomas were derived from thrombi they should also be found in the ventricles. It is very unlikely that a thrombus several centimetres in diameter would ever organize completely and none of the numerous thrombi, in auricle or ventricle, examined during the preparation of this paper have borne the slightest resemblance to a myxoma. Myxomas, unlike thrombi, are not associated with rheumatic or other inflammatory lesions of the endocardium and the slight thickening of the valve cusps and the chordae tendineae noted in the present series, and previously described by Dexter and Work (1941) in one case, may be attributed to the stress imposed by the mechanically induced deformity of the valve. Ribbert (1924) believed that a neoplasm might arise in myxoid embryonic remnants in the region of the fossa ovalis. Rezek (1938) described an entirely intramural nodule of the right auricle containing epithelial cells and cysts which might well be an early myxoma or at least a complex malformation in which neoplasia might occur. Prichard (1951) has assessed this theory by routine sectioning of the fossa ovalis and although myxoid tissue has not been found he has demonstrated minor endocardial malformations in the form of collections of capillary lacunae lined by swollen endothelial cells. This has been confirmed by personal experience but the malformations so far detected are even smaller than those illustrated by Prichard. However, it is clear that minute foci of hyperplasia do exist in this region.

In the literature the relationship of the tumour and its contained elastic tissue to the elastic lamina of the auricle has either been disregarded or it is merely stated that the elastic lamina of the auricle was intact. All of the angiomyxomas here considered showed a definite elastic lamina close to the surface and in three of them this could be traced to its junction with the auricular elastic fibres, and in Case 1 the hypertrophied auricular elastic tissue split to enclose the tumour. In three the auricular elastic tissue beneath the tumour was diminished. These findings indicate that the tumour originated within the wall of the auricle and, by its expansion, carried with it into the cavity a proportion of the superficial auricular elastic fibres.

The occurrence of neoplasia in malformations is well established and it is suggested that these tumours grow, during adult life, from elements of small pre-existing hamartomas. A comparable process may occur in epidermal moles. They are essentially angiomatous and whether or not they should be called fibromas, myxomas, or endotheliomas is irrelevant if they are regarded as an overgrowth, in varying proportions, of constituents which are normal at some stage in the development of the part. Whether or not they should be considered to be true neoplasms is a matter of personal belief. If anomalous differentiation can be synonymous with neoplasia then the cardiac angiomyxoma is a tumour.

I conclude with what may be the earliest descrip- $\stackrel{\mathbb{Q}}{\varrho}$ tion of one of these tumours, cited by King (1845) क from the notes of Thomas Hodgkin. The subject $\vec{\circ}$ was a woman aged 55 .

"In the left auricle, close to the margin of the $\vec{\omega}$ foramen ovale, was attached a large polypiform body of about the size of a pullet's egg. The sur- 용 face by which it was attached was nearly the size or of a shilling. Though rather dark and discoloured t in some parts, it was generally of a light yellow, $\omega$ and semi-transparent, with some opaque white $\mathscr{C}_{0}$ specks dispersed through it, having some resem- 0 blance to the opaque points in soft soap, but rarer $\frac{}{J}$ and smaller. It was firmer than the ordinary fibrin- ous concretions which are found in the heart, and $Z$ was especially so about its root. On fine injection being thrown into the coronary arteries, minute $\frac{\mathbb{D}}{3}$ vessels were seen beautifully ramifying through the $\frac{3}{\sigma}$ transparent substance. This polypiform concretion 음 was covered by a thin membrane continuous with that of the lining membrane of the auricle. Though $\overrightarrow{0}$ its substance was not disposed in layers, some N appearances in it (as, for instance, darkish poines of a brownish colour) had a trace of arrangememt equi-distant from the surface."

\section{Summary}

Four tumours arising in the left auricle near to $\frac{0}{8}$ the fossa ovalis are described. They can be placed in a sequence ranging from almost pure $\overline{0}$ "myxoma" to typical haemangioma. It is considered, upon histological grounds, that they arise from elements of pre-existing intramural hamartomas. As they exhibit slight but definite powers of growth in excess of those of the normal struc-: tures they should be termed hamartoblastomas and 3 . may for convenience be described as angio- $\dot{0}$ myxomas, although the term myxoma, which indicates a peculiar feature of the collagenous 0 stroma, probably in part degenerative, has little merit.

I am indebted to Mr. G. W. Moore for the photographs.

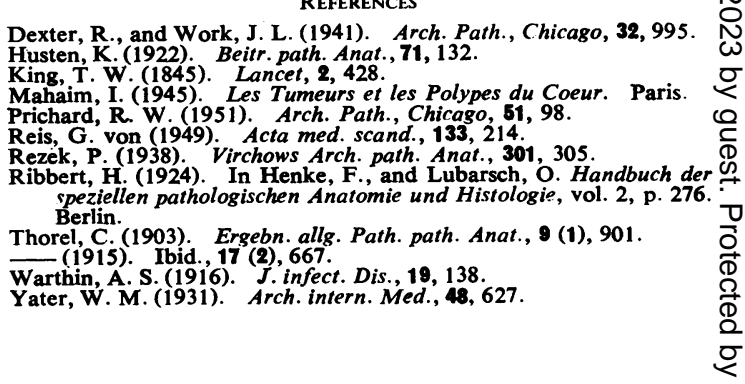

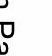

